\title{
BeppoSAX spectroscopy of the luminous X-ray sources in M 33
}

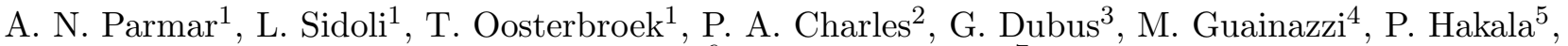 \\ W. Pietsch ${ }^{6}$, and G. Trinchieri ${ }^{7}$ \\ 1 Astrophysics Division, Space Science Department of ESA, ESTEC, Postbus 299, 2200 AG Noordwijk, \\ The Netherlands \\ 2 Dept of Physics \& Astronomy, University of Southampton, Southampton, Hants, SO17 1BJ, UK \\ 3 Astronomical Institute "Anton Pannekoek", Kruislaan 403, 1098 SJ Amsterdam, The Netherlands \\ 4 XMM-Newton SOC, ESA Villafranca, Apartado 50727, 28080, Madrid Spain \\ ${ }^{5}$ Observatory \& Astrophysics Lab., University of Helsinki, Helsinki, Finland \\ 6 Max-Planck-Institut für Extraterrestrische Physik, 85740 Garching bei München, Germany \\ 7 Osservatorio Astronomico di Brera, Via Bianchi 46, Merate 22055, Italy
}

Received 16 October 2000 / Accepted 18 December 2000

\begin{abstract}
The nearby galaxy M 33 was observed by the imaging X-ray instruments on-board BeppoSAX. Two observations at different phases of the 105.9 day intensity cycle of the luminous central source X-8 failed to reveal the expected modulation, suggesting that it is probably transitory. Similar behavior has been observed from several X-ray binary sources. This strengthens somewhat the idea that M 33 X-8 is a black hole accreting from a binary companion. The $0.2-10 \mathrm{keV}$ spectrum of M $33 \mathrm{X}-8$ can best be modeled by an absorbed power-law with a photon index, $\alpha$, of $1.89 \pm{ }_{0.79}^{0.40}$ and a disk-blackbody with a temperature, $k T$, of $1.10 \pm 0.05 \mathrm{keV}$ and a projected inner-disk radius of $55.4 \pm{ }_{7.7}^{6.0} \mathrm{~km}$. This spectral shape is in good agreement with earlier ASCA results. The 2-10 keV spectra of M 33 X-4, X-5, X-7, X-9 and X-10 are all consistent with power-law or bremsstrahlung models, whilst that of X-6 appears to be significantly more complex and may be reasonably well modeled with a disk-blackbody with $k T=1.7 \pm 0.2 \mathrm{keV}$ and a projected inner-disk radius of $7 \pm 2 \mathrm{~km}$. The spectrum of M $33 \mathrm{X}-9$ is rather hard with $\alpha=1.3$. Compared to earlier Einstein and ROSAT observations, M 33 X-7 and X-9 varied in intensity, M 33 X-4, X-6, and X-10 may have varied and M 33 X-5 remained constant.
\end{abstract}

Key words. galaxies: individual (M 33) - galaxies: nuclei - X-ray: galaxies

\section{Introduction}

M 33 (NGC 598) is one of the nearest galaxies (795 kpc, van den Bergh 1991) and is classified as a late-type Sc spiral. As well as containing several point X-ray sources, M 33 is unique amongst the Local Group in having a very bright $\left(\sim 10^{39} \mathrm{erg} \mathrm{s}^{-1}\right)$ X-ray source (X-8) located at a position consistent (to within the positional error of the Einstein High Resolution Imager (HRI) of $3^{\prime \prime}$ ) with the optical nucleus of the galaxy (see Long et al. 1981; Markert \& Rallis 1983; Gottwald et al. 1987; Trinchieri et al. 1988; Dubus et al. 1997). This source dominates the X-ray flux from the galaxy, being responsible for $\sim 70 \%$ of the $0.15-4.5 \mathrm{keV}$ emission. Another X-ray source in M 33 (X-7) is an eclipsing X-ray binary pulsar (Peres et al. 1989; Schulman et al. 1993, 1994; Larson \& Schulman 1997) with a 3.45 day orbital period and a $0.31 \mathrm{~s}$ pulse period (Dubus et al. 1999).

Send offprint requests to: A. N. Parmar, e-mail: aparmar@astro.estec.esa.nl
The X-ray sources in M 33 have been studied by Einstein, EXOSAT, ROSAT, and ASCA, as well as by earlier nonimaging instruments. With an exposure of $50 \mathrm{ks}$ ROSAT Position Sensitive Proportional Counter (PSPC) observations of M 33 have detected 35 sources in the central $30^{\prime}$ which are brighter than $10^{36} \mathrm{erg} \mathrm{s}^{-1}$ (Long et al. 1996, see also Haberl \& Pietsch 2000). From an examination of archival ROSAT data spanning over 6 years (a total of $387 \mathrm{ks}$ of HRI time and $62 \mathrm{ks}$ of PSPC time), Dubus et al. (1997) find that the X-ray intensity of M 33 X-8 is almost certainly modulated with a period of $105.9 \pm 0.1$ days.

The nature of $\mathrm{M} 33 \mathrm{X}-8$ is unclear. X-ray transients such as Aql X-1 and 4U-1630-47 show outbursts on timescales of hundreds of days, but are not periodic (e.g., Kuulkers et al. 1997) while a number of persistent X-ray sources exhibit long term periodicities in their X-ray intensities. Extended observations using the AllSky Monitor (ASM) on the Rossi X-ray Timing Explorer 
Table 1. BeppoSAX M 33 observing log. Phase, $\Phi$, is determined using the 105.9 day ephemeris of Dubus et al. (1997) where the M 33 X-8 maximum is expected at phase 0.0 and minimum at $\sim 0.7$

\begin{tabular}{|c|c|c|c|c|c|c|c|}
\hline \multirow[t]{2}{*}{ Num. } & \multicolumn{2}{|c|}{ Observation } & \multicolumn{2}{|c|}{ Exposure } & \multicolumn{2}{|c|}{ M 33 X-8 count rate } & \multirow[t]{2}{*}{$\Phi$} \\
\hline & $\begin{array}{c}\text { Start } \\
\text { (year mn dy hr:mn) }\end{array}$ & $\begin{array}{c}\text { End } \\
(\mathrm{mn} \text { dy hr:mn) }\end{array}$ & $\begin{array}{r}\text { LECS } \\
(\mathrm{ks})\end{array}$ & $\begin{array}{r}\text { MECS } \\
(\mathrm{ks})\end{array}$ & $\begin{array}{c}\text { LECS } \\
\left(0.1-2 \mathrm{keV} ; \mathrm{s}^{-1}\right)\end{array}$ & $\begin{array}{c}\text { MECS } \\
\left(1.8-10 \mathrm{keV} ; \mathrm{s}^{-1}\right)\end{array}$ & \\
\hline 1 & 1998 Aug. 24 13:05 & Aug. 25 22:26 & 32.3 & 59.9 & $0.068 \pm 0.002$ & $0.157 \pm 0.002$ & 0.72 \\
\hline 2 & 1999 Jan. 06 20:16 & Jan. 07 18:38 & 0.9 & 1.1 & $0.045 \pm 0.008$ & $0.131 \pm 0.011$ & 0.98 \\
\hline 3 & 2000 Jul. 09 17:56 & Jul. 13 04:33 & 7.3 & 37.4 & $0.065 \pm 0.004$ & $0.141 \pm 0.002$ & 0.20 \\
\hline
\end{tabular}

(R-XTE) have revealed changes in some long term periodicities (e.g., Paul et al. 2000); Wojdowski et al. 1998; Kong et al. 1998). The detection of a 106 day periodicity over $\sim 20$ cycles implies a regularity and duty cycle incompatible with typical soft X-ray transients. M 33 X-8 may instead be a moderate mass black hole. Until recently, the M 33 nuclear source was frequently considered (as are many of the bright sources in normal galaxies that exceed the Eddington limit of $\sim 10^{38} \mathrm{erg} \mathrm{s}^{-1}$ for a $1 M_{\odot}$ compact object) to be a "mini-" (or perhaps "micro-") active galactic nucleus (AGN). However, the X-ray spectrum of X-8 appears to be much steeper than a classical AGN powerlaw and can be well fit by a multi-color disk blackbody model plus a harder power-law (Takano et al. 1994). This spectral shape is characteristic of the galactic black hole candidates which is often ultra-soft at low-energies and ultra-hard at high-energies. The ultra-soft component may originate from an optically thick accretion disk, while the ultra-hard component may result from comptonization of soft photons by hot electrons (Sunyaev \& Trümper 1979). The accretion disk model is parameterized by only two quantities, the projected inner disk radius, $r_{\text {in }}(\cos i)^{0.5}$, and the temperature at this inner radius, $k T_{\text {in }}$ (Mitsuda et al. 1984; Makishima et al. 1986). Using ASCA, Takano et al. (1994) obtain a value for $r_{\text {in }}(\cos i)^{0.5}$ of $\sim 50 \mathrm{~km}$, typical of black hole candidates in their high state. At the distance of M 33, this corresponds to a black hole mass of $\sim 10 M_{\odot}$.

In this paper we report on BeppoSAX observations of M 33 primarily designed to investigate any spectral changes associated with the 105.9 day cycle of M 33 X-8 (Sect. 3) as well as the spectral and temporal properties of the luminous X-ray sources in M 33 (Sect. 4).

\section{Observations}

Data from the coaligned Low-Energy Concentrator Spectrometer (LECS; 0.1-10 keV; Parmar et al. 1997), Medium-Energy Concentrator Spectrometer (MECS; 1.8$10 \mathrm{keV}$; Boella et al. 1997), and the Phoswich Detection System (PDS; 15-300 keV; Frontera et al. 1997) on-board BeppoSAX are presented. The MECS consists of two identical grazing incidence telescopes with imaging gas scintillation proportional counters in their focal planes. The LECS uses an identical concentrator system as the MECS, but utilizes an ultra-thin entrance window and a driftless configuration to extend the low-energy response to
$0.1 \mathrm{keV}$. The fields of view (FOV) of the LECS and MECS are circular with diameters of $37^{\prime}$ and $56^{\prime}$, respectively. The MECS has a $2^{\prime}$ thick circular window support structure which is centered $10^{\prime}$ from the FOV center. In addition, four $2^{\prime}$ thick radial spokes extend outwards from the circular support. The non-imaging PDS consists of four independent units arranged in pairs each having a separate collimator. Each collimator was alternatively rocked on- and $210^{\prime}$ off-source every $96 \mathrm{~s}$ during the observations.

M 33 was observed 3 times by BeppoSAX (see Table 1). The observing times were chosen in order to study M 33 X8 near the maximum and minimum of the 105.9 day X-ray cycle. The maxima occur at $\mathrm{MJD}=48750.3+n$ 105.9, and the minima approximately 0.7 in phase later (Dubus et al. 1997). The 1998 August observation was close to the 105.9 day minimum $(\Phi=0.72)$, and the 1999 January observation close to the maximum $(\Phi=0.98)$. However, this observation was curtailed due to technical problems and repeated in 2000 July at $\Phi=0.20$, still close to the expected maximum of the 105.9 day cycle. Good data were selected from intervals when the elevation angle above the Earth's limb was $>5^{\circ}$ and when the instrument configurations were nominal, using the SAXDAS 2.0.0 data analysis package.

Figure 1 shows the MECS $1.8-10 \mathrm{keV}$ image obtained by summing data from all 3 observations. The total exposure is $98.3 \mathrm{ks}$. For clarity, the data have been rebinned by a factor 2 into $16^{\prime \prime}$ pixels. No corrections for vignetting or obscuration by the MECS window support structure (see above) have been applied. As well as the central M 33 X-8 source which dominates the overall X-ray flux, M 33 X-4, X-5, X-6, X-7, X-9 and X-10 are clearly detected. The luminous source M $33 \mathrm{X}-3$ is strongly obscured by the MECS strongback and was not detected. In addition, the source identified here as M $33 \mathrm{X}-9$ is resolved into 3 sources in ROSAT and Einstein observations (Long et al. 1996; Peres et al. 1989).

\section{M $33 \times-8$ analysis}

LECS and MECS data were extracted centered on the position of M 33 X-8 using radii of $6^{\prime}$ and $4^{\prime}$, respectively. This LECS extraction radius is smaller than normally used in order to minimize the contribution from nearby sources. The cosmic and internal instrument backgrounds are $\sim 5 \%$ of the source flux, and so background subtraction is not critical and was performed using source free regions of 


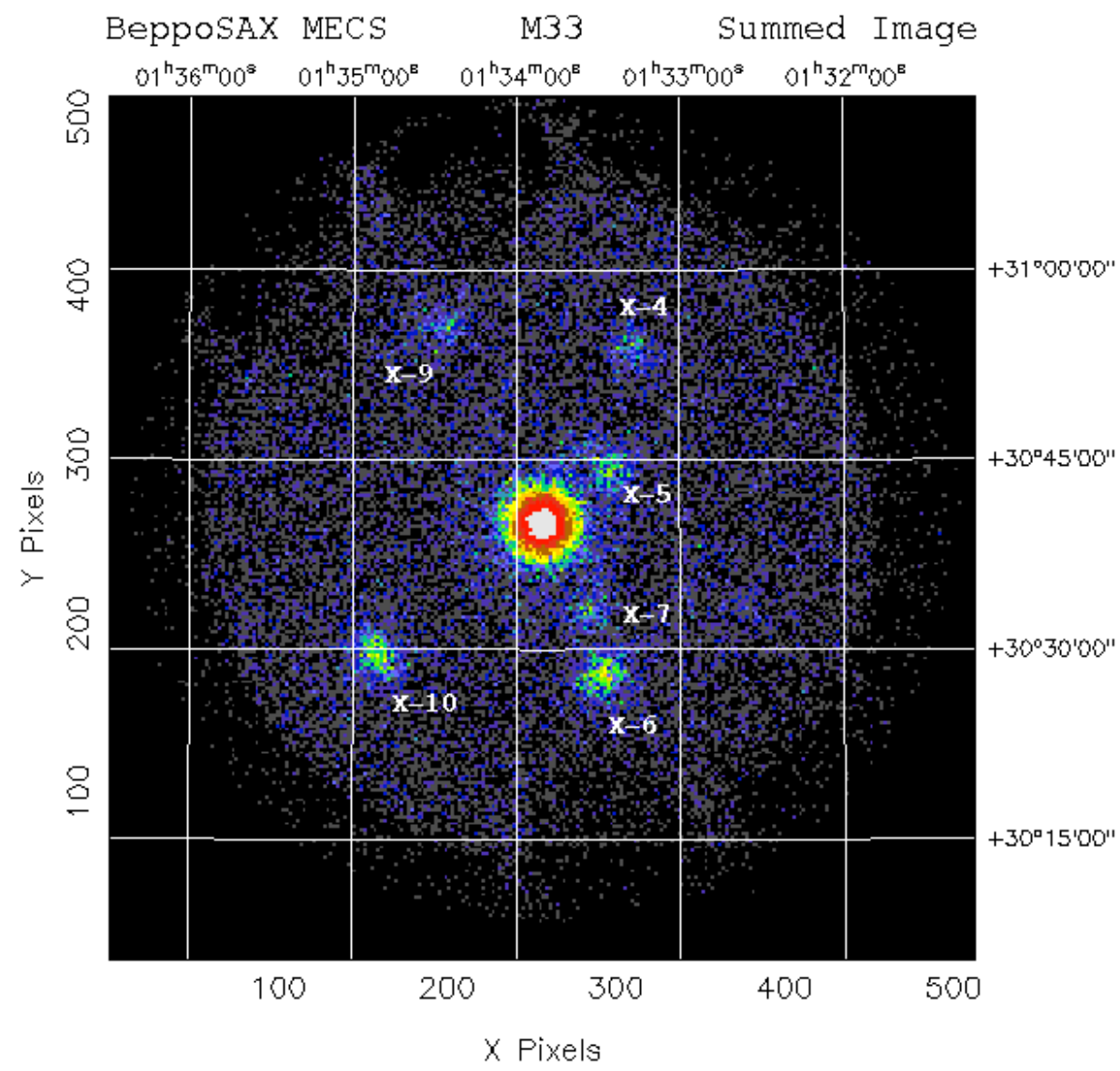

Fig. 1. MECS $1.8-10.0 \mathrm{keV}$ image of M 33 obtained by summing data from all 3 observations. M 33 X- 8 is the central bright source. The data have been rebinned by a factor 2 into $16^{\prime \prime}$ pixels. The location of the sources discussed in Sect. 4 are indicated. The "cut-outs" at the top and bottom of the image are due to the removal of instrument calibration source events
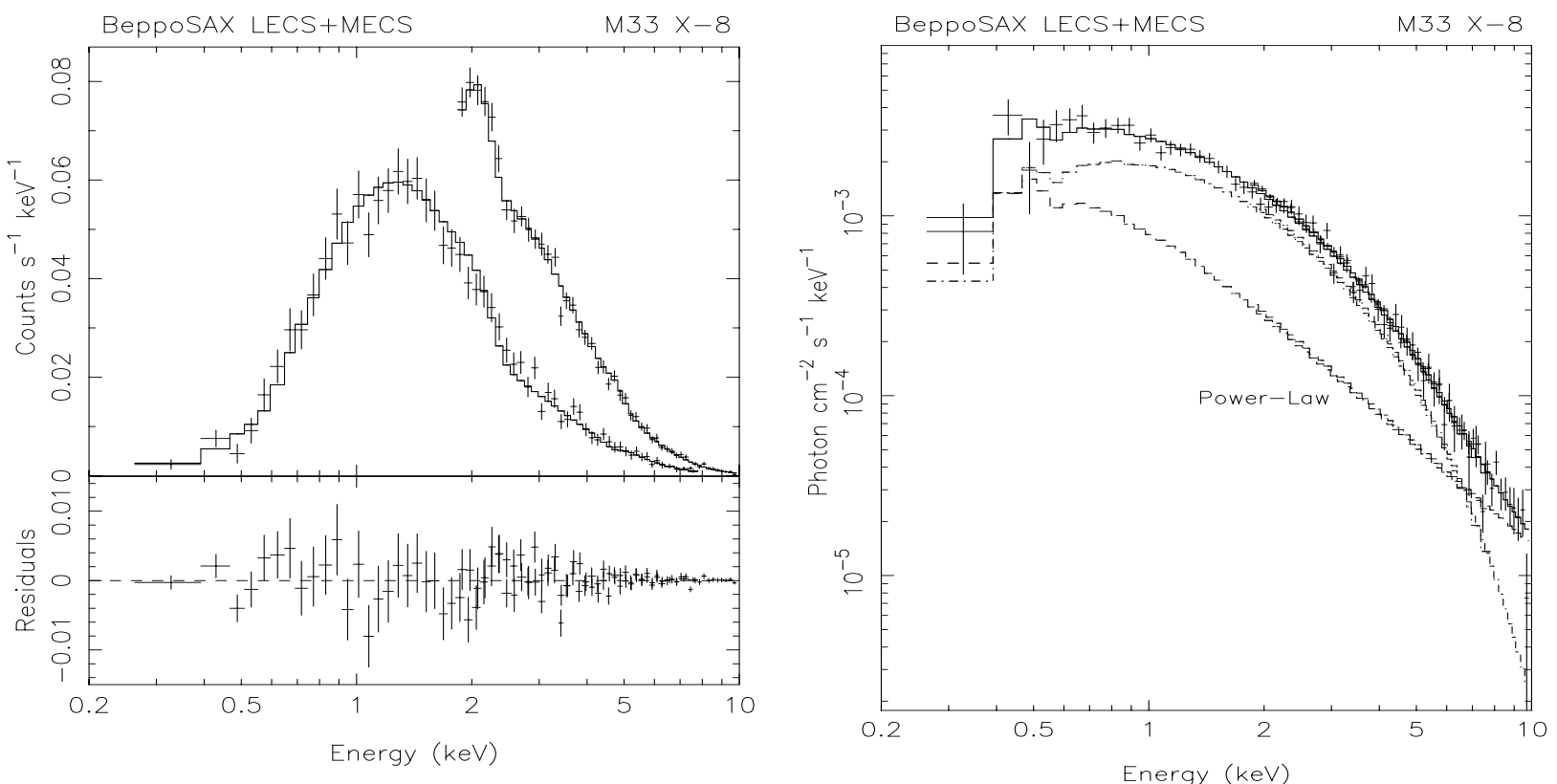

Fig. 2. The M 33 X-8 LECS and MECS count spectrum and residuals when the power-law and disk-blackbody model is fit to the summed spectrum (left panel). The residuals are given in count $\mathrm{s}^{-1} \mathrm{keV}^{-1}$. The right panel shows the photon spectrum with the contribution of the power-law indicated 
Table 2. MECS positions (J2000) of the faint point sources ( $\sim 1^{\prime}$ uncertainty radius), compared with the ROSAT PSPC positions reported in Long et al. (1996). Source X-9 is unresolved in the MECS observations, but resolved into three source (a, b, c) with ROSAT and Einstein. For X-9 (Long et al. 1981) and X-9(c) (Peres et al. 1989) the Einstein coordinates are given. B74 = Boulesteix et al. 1974 catalog of M 33 H II regions; L81 = Long et al. 1981; L96 = Long et al. 1996; CS82a = Christian \& Schommer 1982a; CS82b = Christian \& Schommer 1982b

\begin{tabular}{lccccl}
\hline Source & $\begin{array}{c}\text { RA (MECS) } \\
(\mathrm{h} \mathrm{m} \mathrm{s})\end{array}$ & $\begin{array}{c}\text { Dec. (MECS) } \\
\left({ }^{\circ}{ }^{\prime \prime \prime}\right)\end{array}$ & $\begin{array}{c}\text { RA (PSPC) } \\
(\mathrm{h} \mathrm{m} \mathrm{s})\end{array}$ & $\begin{array}{c}\text { Dec. (PSPC) } \\
\left({ }^{\circ}{ }^{\prime \prime \prime}\right)\end{array}$ & Notes \\
\hline $\mathrm{X}-4$ & 013317.5 & +305402.0 & 013314.8 & +305328.5 & SNR 136+396 (L96); H II IC133 (L81) \\
$\mathrm{X}-5$ & 013325.9 & +304433.8 & 013324.3 & +304404.7 & $\ldots$ \\
$\mathrm{X}-6$ & 013327.7 & +302812.5 & 013327.5 & +302722.7 & Globular cluster? (L81; CS82a) \\
$\mathrm{X}-7$ & 013331.8 & +303336.4 & 013333.8 & +303212.8 & X-ray eclipsing binary; H II B208 (B74; L81) \\
$\mathrm{X}-9$ & 013426.0 & +305559.7 & 013436.3 & +305500.4 & Einstein position (L81) \\
$\mathrm{X}-9(\mathrm{a})$ & $\ldots$ & $\ldots$ & 013438.7 & +305510.3 & $\ldots$ \\
$\mathrm{X}-9(\mathrm{~b})$ & $\ldots$ & $\ldots$ & 013425.5 & +305524.5 & Globular cluster? (n.3/4 in CS82b) \\
$\mathrm{X}-9(\mathrm{c})$ & $\ldots$ & $\ldots$ & 013431.5 & +305620.5 & Elliptical galaxy at $z=0.03 ?$ (CS82a) \\
$\mathrm{X}-10$ & 013450.8 & +302917.3 & 013451.7 & +302905.9 & $\ldots$ \\
\hline
\end{tabular}

sky. Table 1 gives the $0.1-2.0 \mathrm{keV}$ (LECS) and 2.0-10 keV (MECS) count rates for each observation.

The M 33 X-8 spectra were investigated by simultaneously fitting data from the LECS and MECS instruments. All spectra were rebinned to oversample the full width half maximum of the energy resolution by a factor 3 and to have additionally a minimum of 20 counts per bin to allow use of the $\chi^{2}$ statistic. Data were selected in the energy ranges $0.2-8.0 \mathrm{keV}$ (LECS) and 1.8-10 keV (MECS) where the instrument responses are well determined and sufficient counts obtained. The photoelectric absorption cross sections of Morrison \& McCammon (1983) are used throughout. Factors were included in the spectral fitting to allow for normalization uncertainties between the two instruments.

\subsection{August spectrum}

Initially, simple models were tried, including absorbed power-law, thermal bremsstrahlung and cutoff powerlaw $\left(E^{-\alpha} \exp -\left(E_{\mathrm{co}} / k T\right)\right)$ models. The power-law and bremsstrahlung models give unacceptable fits with $\chi^{2}$ of 380.8 and 149.8 for 101 degrees of freedom (dof), respectively. The cutoff power-law model fit is acceptable with a $\chi^{2}$ of 118.1 for 100 dof. Next, more complex models consisting of a power-law and a blackbody and a power-law and a disk-blackbody were fit. These both give acceptable fits with $\chi^{2}$ s of 106.0 and 108.9 for 99 dof, respectively. The best-fit inner disk radius is $55.2 \pm 7.6 \mathrm{~km}$ for a distance of $795 \mathrm{kpc}$ and a disk inclination of $0^{\circ}$. The fit results are summarized in Table 4 . The absorption corrected $1.0-10 \mathrm{keV}$ source luminosity is $1.310^{39} \mathrm{erg} \mathrm{s}^{-1}$ for a distance of $795 \mathrm{kpc}$.

\subsection{July spectrum}

The spectra were created in the same way as for the 1998 August observation and the same spectral models applied. The results are given in Table 4 . The exposure times for this observation are shorter than for the 1988 August observation, and the spectrum can be successfully modeled using either bremsstrahlung, cutoff power-law, power-law and blackbody or power-law and disk-blackbody models. The derived spectral parameters are consistent with those from the earlier observation with an inner disk radius of $50.0 \pm \frac{13.2}{22.6} \mathrm{~km}$ for the same assumptions as above. The absorption corrected $1.0-10 \mathrm{keV}$ source luminosity is $1.210^{39} \mathrm{erg} \mathrm{s}^{-1}$ for a distance of $795 \mathrm{kpc}$.

\subsection{Combined spectrum}

Since the spectral fit parameters derived from the fits to the 1998 August and 2000 July data are consistent, these data were combined to produce a "summed" spectrum. Data from the short (MECS exposure of $1.1 \mathrm{ks}$ ) observation in 1999 January were also included. The same spectral models as above were fit and the results summarized in Table 4. As found by Takano et al. (1994), the bestfit model is the power-law and disk-blackbody combination with a $k T_{\text {in }}$ of $1.10 \pm 0.05 \mathrm{keV}$ and an inner radius of $55.4 \pm{ }_{7.7}^{6.0} \mathrm{~km}$ for a distance of $795 \mathrm{kpc}$ and a disk inclination angle of $0^{\circ}$. The mean, absorption corrected, $1.0-10 \mathrm{keV}$ luminosity of M $33 \mathrm{X}-8$ is $1.2510^{39} \mathrm{erg} \mathrm{s}^{-1}$ for a distance of $795 \mathrm{kpc}$. The $90 \%$ confidence upper limit to a narrow emission line at $6.5 \mathrm{keV}$ is $100 \mathrm{eV}$.

The galactic absorption in the direction of M $33 \mathrm{X}-8$ is $5.610^{20}$ atom $\mathrm{cm}^{-2}$ (Dickey \& Lockman 1990). Using the power-law and disk-blackbody model, the best fit absorption of $\left(1.2 \pm{ }_{0.6}^{1.0}\right) \quad 10^{21}$ atom $\mathrm{cm}^{-2}$ suggests the presence of a small amount of additional absorption. The $3 \sigma$ upper-limit to any PDS counts in the $15-40 \mathrm{keV}$ band is $0.06 \mathrm{~s}^{-1}$, consistent with the extrapolation of the bestfit power-law and disk-blackbody spectrum to this energy range which gives 0.02 count $\mathrm{s}^{-1}$. For a power-law spectrum with $\alpha=2.1$ the upper-limit corresponds to a flux of $<0.4 \mathrm{mCrab}$. 

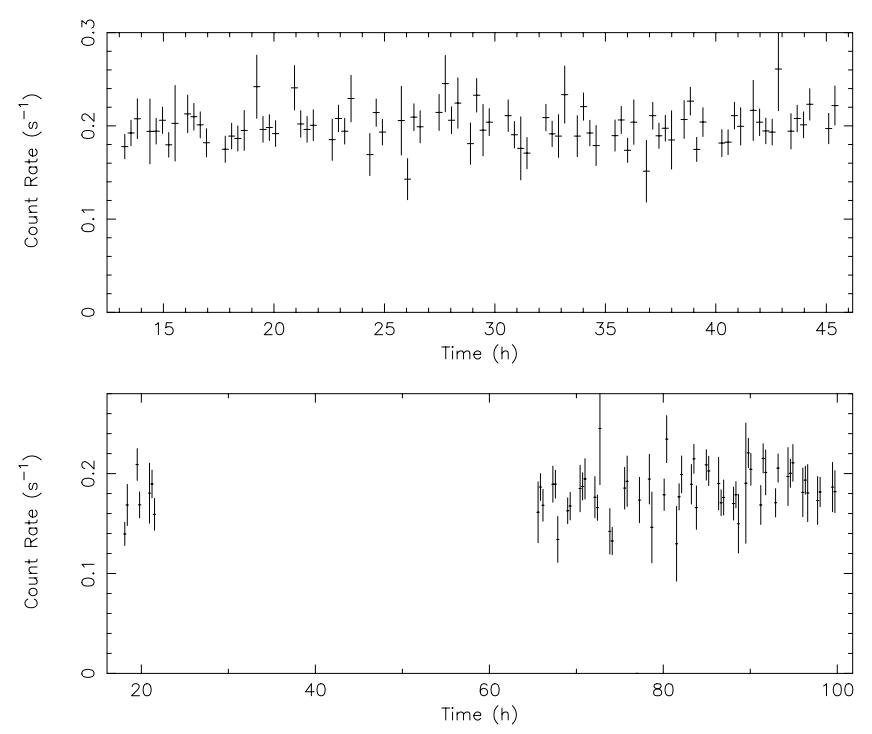

Fig. 3. MECS $2-10 \mathrm{keV}$ lightcurves for M $33 \mathrm{X}-8$ with a binning of $1024 \mathrm{~s}$ for the 1998 August (upper panel) and 2000 July (lower panel) observations. No background has been subtracted

\subsection{Time variability}

Figure 3 shows $2-10 \mathrm{keV}$ MECS lightcurves of M 33 X-8 during 1998 August and 2000 July observations with a binning time of $1024 \mathrm{~s}$. The LECS and MECS data from these observations were searched for the presence of periodic signals. None were found. During the 1998 August observation the $3 \sigma$ limit to any $2-10 \mathrm{keV}$ (MECS) root mean square variability is $<7.6 \%$ when evaluated on a timescale of $3000 \mathrm{~s}$. In the energy band $0.1-2.0 \mathrm{keV}$ (LECS) the upper limit evaluated in the same way is $<5.3 \%$. For the 2000 July observation the corresponding upper limits are $<25 \%$ and $<29 \%$.

The mean ROSAT HRI count rate is $0.20 \mathrm{~s}^{-1}$ and the modulation appears sinusoidal-like with an amplitude of $\sim 20 \%$. The folded $0.1-2.5 \mathrm{keV}$ ROSAT lightcurve (see Figs. 3 and 4 of Dubus et al. 1997) has a mean count rate of $0.20 \mathrm{~s}^{-1}$ with a sinusoidal-like modulation superposed with an amplitude of $\sim 20 \%$. This implies that the count rates of the 1999 January and 2000 July observations should be $\sim 40 \%$ higher than that of 1998 August, should the 105.9 day modulation be present. However, the MECS 2.0-10.0 keV count rate at $\Phi=0.72$ (close to the expected minimum of the cycle) is higher than at the expected maximum. In order to compare our BeppoSAX results for the two long observations with those of Dubus et al. (1997), we folded the best-fit BeppoSAX power-law and disk-blackbody models (see Table 4) through the HRI onaxis response matrix obtained from the HEASARC. This gives predicted HRI count rates of $0.221 \pm 0.007 \mathrm{~s}^{-1}$ and $0.195 \pm 0.012 \mathrm{~s}^{-1}$ for the 1998 August and 2000 July observations, respectively. These points are shown together with the HRI results of Dubus et al. (1997) in Fig. 4. The derived count rates do not support the continued presence of the 105.9 day modulation, although we cannot

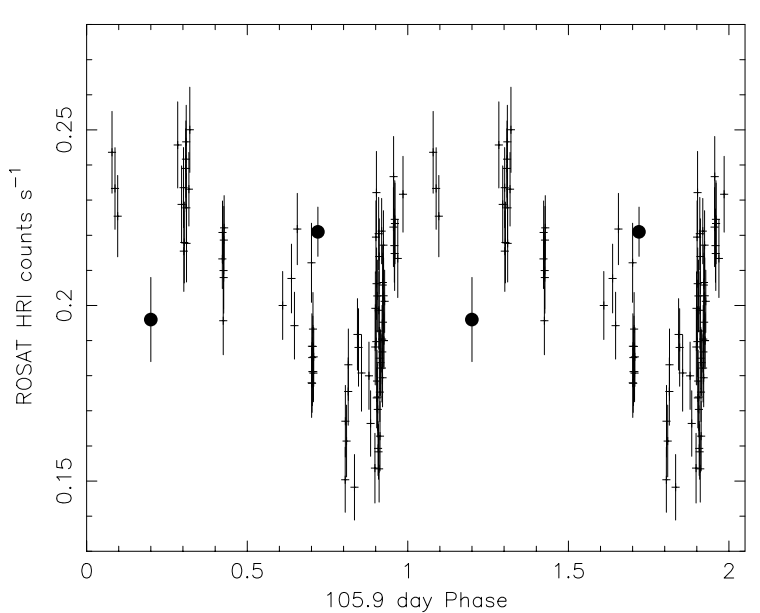

Fig. 4. The ROSAT HRI count rates of Dubus et al. (1997) folded over the 105.9 day period. The count rates corresponding to the two BeppoSAX measurements are indicated with filled circles

Table 3. Point sources detected in the MECS. $C$ is the average MECS $1.8-10 \mathrm{keV}$ background subtracted count rate. $R$ is the extraction radius used for the MECS spectral analysis and $\theta$ the source off-axis angle. Note that the MECS strongback obscures sources located between $\theta=9-11^{\prime} . V$ is the approximate vignetting correction to correct $C$ to the on-axis case

\begin{tabular}{lclrl}
\hline Source & $\begin{array}{c}C \\
\left(10^{-3} \mathrm{~s}^{-1}\right)\end{array}$ & $\begin{array}{l}R \\
\left({ }^{\prime}\right)\end{array}$ & $\begin{array}{r}\theta \\
\left({ }^{\prime}\right)\end{array}$ & $V$ \\
\hline $\mathrm{X}-4$ & $2.61 \pm 0.26$ & 4 & 16 & 2.4 \\
$\mathrm{X}-5$ & $3.40 \pm 0.26$ & 2 & 6 & 1.3 \\
$\mathrm{X}-6$ & $6.15 \pm 0.29$ & 2 & 12 & 1.9 \\
$\mathrm{X}-7$ & $2.07 \pm 0.19$ & 2 & 7 & 1.3 \\
$\mathrm{X}-8$ & $151.8 \pm 1.3$ & 4 & 0 & 1.0 \\
$\mathrm{X}-9$ & $2.85 \pm 0.27$ & 4 & 18 & 2.7 \\
$\mathrm{X}-10$ & $8.95 \pm 0.40$ & 4 & 17 & 2.5 \\
\hline
\end{tabular}

entirely exclude the possibility that the modulation is being masked by a higher than usual level of intrinsic variability.

\section{Point sources}

In order to investigate the properties of the other sources identified in Fig. 1 MECS data from all 3 observations were used. Except for M 33 X-6 LECS data were not used since there were too few counts present. During the 1998 August observation M 33 X-4 was partially obscured by a MECS strongback radial spoke (see Sect. 4.2) and these data were excluded from the analysis. For all the other sources no obscuration problems were present. Spectra were extracted centered on each source using the extraction radii listed in Table 3 and $2^{\prime}$ in the case of the LECS M 33 X-6 data. These radii were chosen to minimize contamination from nearby sources and take into account the position of the MECS window support strongback. The spectra were rebinned in the same way as in Sect. 2 
and data selected in the energy ranges where sufficient counts were obtained. Since these sources are all faint by BeppoSAX standards care was taken to reliably estimate the background. In order to properly take into account all the possible contaminating contributions, especially from the bright nuclear source M 33 X-8, local backgrounds, each extracted from the same off-axis angle as the corresponding source, but at the geometrically opposite position in the FOV, were used. This is also necessary because the MECS instrument background has a component due to $\mathrm{Mn} \mathrm{K} \alpha$ calibration source $\mathrm{X}$-rays which are detected within the FOV. This effect is most noticeable close to the calibration sources and in particular for M 33 X-9. A comparison of these local backgrounds with the average properties of background taken from high latitude pointings reveals that no strong evidence for diffuse emission is present in the MECS M 33 data. A higher local background $(\sim 20 \%)$ is present around M $33 \mathrm{X}-8$, but this is consistent with being due to the X-ray mirror scattering wings.

Instrument response files appropriate to the off-axis positions of each source were used, except for M 33 X-5 and $\mathrm{X}-7$, which are close enough to the on-axis position to use the standard response files.

\subsection{Spectral fit results}

Absorbed power-law and bremsstrahlung models were first fit to each of the spectra and the results presented in Table 5. Fits were repeated with the absorption constrained to be greater than, and equal to, the galactic value for each source. With the exception of M 33 X-6 (and possibly M $33 \mathrm{X}-10$ ) these models give reasonable fits to all the spectra and it is not possible to exclude either model. In the case of M 33 X-6, the best-fit absorbed power-law and bremsstrahlung models give $\chi^{2}$ 's of 51.7 and 44.2 for 28 dof, respectively. The best-fit to the M 33 X-6 spectrum is instead obtained with an absorbed disk-blackbody model with $k T_{\text {in }}=1.7 \pm 0.2 \mathrm{keV}$ and a normalization $\left(r_{\text {in }}(\cos i)^{0.5}\right)$ of $7 \pm 2 \mathrm{~km}$. The $90 \%$ confidence upper limit to a narrow emission line at $6.5 \mathrm{keV}$ from M $33 \mathrm{X}-6$ is $540 \mathrm{eV}$. In the case of M $33 \mathrm{X}-10$, a broad feature appears to be present at $\sim 4 \mathrm{keV}$. This can be modeled by the addition of a $(\sigma=200 \mathrm{eV})$ Gaussian line to the best-fit power-law to give a $\chi^{2}$ of 18.6 for 15 dof. The line energy is $4.2 \pm{ }_{0.2}^{0.3} \mathrm{keV}$ and the equivalent width $440 \mathrm{eV}$.

Table 5 includes estimates of the source luminosities extrapolated (when necessary) to the $2-10 \mathrm{keV}$ energy range. Photon spectra, deconvolved using the best-fit power-law models are shown in Fig. 5, except for M 33 X-6 where the best-fit disk-blackbody model is used. With the exception of M 33 X-9 and X-10, all the sources appear to be relatively soft in the $2-10 \mathrm{keV}$ energy range.

\subsection{Time variability}

In order to investigate if any of the point sources exhibit excess variability, a Kolmogorov-Smirnov test as implemented in the FTOOLs package LCSTAT was performed on each of the lightcurves presented in Figs. 6 and 7. The sources M 33 X-4, X-5, and X-10 show some evidence for excess variability on time scales $>1024 \mathrm{~s}$ with probabilities for being constant of $4.8 \%, 4.4 \%$, and $5.4 \%$ for M $33 \mathrm{X}$ 4, (obs. 1), X-5 (obs. 3), and X-10 (obs. 1), respectively. The other 3 sources do not show evidence for variability during either long observation with probabilities for being constant of $32 \%$ (X-6, obs. 1), $61 \%$ (X-7, obs. 1, >2048 s), and $19 \%$ (X-9, obs. 3). For comparison, data from sourcefree regions of the image were also extracted and processed in the same way. No evidence for any excess variability was found.

A comparison between the 1998 August (Fig. 6), and 2000 July (Fig. 7) observations, does not show evidence for variability, except for M 33 X-4, which showed a factor of $\sim 2$ difference in count rate between the two observations. However, this change is almost certainly due to obscuration by one of the radial spokes of the MECS strongback during the 1998 August observation. The 2000 July observation was made at a different spacecraft roll angle, resulting in the source being well clear of the strongback. The BeppoSAX data are therefore consistent with the association of M $33 \mathrm{X}-4$ with the supernova remnant SNR 136+396 discussed in Long et al. (1996).

Table 6 gives a comparison of the BeppoSAX source intensities with those derived from previous ROSAT PSPC (Long et al. 1996) and Einstein IPC observations (Peres et al. 1989. For M 33 X-4 only the data from the 2000 July observation is used. Unfortunately, due to the lack of good estimates of the absorbing column to most of the sources (see Table 5), it is difficult to reliably extrapolate the BeppoSAX spectra to the PSPC $(0.1-2.4 \mathrm{keV})$ and IPC (0.2-4.5 keV) energy ranges. Instead, we have adopted the spectral model assumed by Long et al. (1996) to convert ROSAT PSPC counts to fluxes. This consists of a powerlaw with $\alpha=2$ and absorption of $10^{21}$ atom $\mathrm{cm}^{-2}$. We note that none of the spectral fits reported in Table 5 are in strong disagreement with such a model. We estimate that the uncertainty in the BeppoSAX fluxes is $\sim 40 \%$, primarily due to uncertainties in instrumental calibration at large off-axis angles.

Examination of Table 6 shows that $\mathrm{M} 33 \mathrm{X}-4$, X-6 (Einstein only), X-7, X-9 and X-10 (ROSAT only) show $>40 \%$ variability with respect to both the Einstein and ROSAT measurements. The source M 33 X-7 shows the strongest evidence for variability being a factor $\sim 3$ fainter during the BeppoSAX observations than previously observed. Since this source exhibits 0.33 day duration eclipses, we verified that the observations were not at the expected times of eclipse using the ephemeris of Larson \& Schulman (1997). Source X-9, not resolved in the MECS image, appears to be variable being a factor $\sim 2.5$ brighter during the BeppoSAX observation than previously, if the ROSAT fluxes of the three separate sources are summed together (the X-9(c) flux is not reported in Long et al. 1996, although it is visible in their figure and appears to be as bright as the other two sources). Since the level of variability of M $33 \mathrm{X}-4, \mathrm{X}-6$, and $\mathrm{X}-10$ is comparable with 
Table 4. M $33 \mathrm{X}-8$ fit results. $N_{\mathrm{H}}$ is the absorption in units of $10^{22}$ atom $\mathrm{cm}^{-2} \cdot r_{\mathrm{in}}(\cos i)^{0.5}$ is in units of km for a distance of 795 kpc. $\alpha$ is the power-law photon index. $90 \%$ confidence limits are given when the $\chi^{2}$ per dof is $<2$

\begin{tabular}{|c|c|c|c|c|c|c|}
\hline Model & $N_{\mathrm{H}}$ & $k T / k T_{\text {in }}(\mathrm{keV})$ & $\alpha$ & $E_{\mathrm{co}}(\mathrm{keV})$ & $r_{\mathrm{in}}(\cos i)^{0.5}$ & $\chi^{2} /$ dof \\
\hline \multicolumn{7}{|l|}{1998 August observation } \\
\hline Power-law & 0.58 & . & 2.7 & $\ldots$ & $\ldots$ & $380.8 / 101$ \\
\hline Bremsstrahlung & $0.24 \pm 0.03$ & $2.93 \pm 0.10$ & $\ldots$ & $\ldots$ & $\ldots$ & $149.8 / 101$ \\
\hline Cutoff power-law & $0.12 \pm 0.04$ & & $0.63 \pm 0.29$ & $1.89 \pm 0.17$ & & $118.1 / 100$ \\
\hline Power-law + blackbody & $0.23 \pm 0.05$ & $0.75 \pm 0.03$ & $2.44 \pm 0.15$ & $\ldots$ & & $106.0 / 99$ \\
\hline Power-law + disk-blackbody & $0.15 \pm 0.06$ & $1.12 \pm 0.06$ & $2.15 \pm{ }_{0.70}^{0.40}$ & $\ldots$ & $55.2 \pm 7.6$ & $108.9 / 99$ \\
\hline \multicolumn{7}{|l|}{2000 July observation } \\
\hline Power-law & $0.55 \pm 0.10$ & & $2.60 \pm 0.07$ & $\cdots$ & $\cdots$ & $105.3 / 71$ \\
\hline Bremsstrahlung & $0.21 \pm 0.06$ & $3.40 \pm 0.20$ & & $\ldots$ & $\ldots$ & $75.4 / 71$ \\
\hline Cutoff power-law & $0.25 \pm 0.10$ & $\ldots$ & $1.49 \pm \begin{array}{l}0.33 \\
0.08\end{array}$ & $3.79 \pm{ }_{0.93}^{1.68}$ & $\ldots$ & $75.2 / 70$ \\
\hline Power-law + blackbody & $0.29 \pm 0.15$ & $0.72 \pm{ }_{0.07}^{0.14}$ & $2.33 \pm 0.20$ & $\ldots$ & $\ldots$ & $76.9 / 69$ \\
\hline Power-law + disk-blackbody & $0.20 \pm 0.20$ & $1.05 \pm \begin{array}{l}0.21 \\
0.10\end{array}$ & $2.06 \pm{ }_{0.73}^{0.51}$ & $\cdots$ & $50.0 \pm 7.7$ & $74.0 / 69$ \\
\hline \multicolumn{7}{|l|}{ All observations } \\
\hline Power-law & 0.55 & & 2.7 & $\ldots$ & $\ldots$ & $396.6 / 105$ \\
\hline Bremsstrahlung & $0.22 \pm 0.03$ & $3.10 \pm 0.09$ & $\ldots$ & $\ldots$ & $\ldots$ & $141.9 / 105$ \\
\hline Cutoff power-law & $0.135 \pm 0.05$ & $\ldots$ & $0.85 \pm 0.18$ & $2.22 \pm{ }_{0.20}^{0.25}$ & $\ldots$ & $125.4 / 104$ \\
\hline Power-law + blackbody & $0.25 \pm 0.05$ & $0.75 \pm 0.03$ & $2.40 \pm 0.12$ & $\ldots$ & $\ldots$ & $122.2 / 103$ \\
\hline Power-law + disk-blackbody & $0.12 \pm \underset{0.06}{0.10}$ & $1.10 \pm 0.05$ & $1.89 \pm{ }_{0.79}^{0.40}$ & $\ldots$ & $55.4 \pm{ }_{7.7}^{6.0}$ & $115.7 / 103$ \\
\hline
\end{tabular}

Table 5. Spectral fit results to the M 33 point sources. The absorption, $N_{\mathrm{H}}$, is in units of $10^{22}$ atom $\mathrm{cm}^{-2}$. Uncertainties are quoted at $90 \%$ confidence. The $2-10 \mathrm{keV}$ absorption corrected source luminosity, $L$, assumes a distance of $795 \mathrm{kpc}$

\begin{tabular}{|c|c|c|c|c|c|c|}
\hline Source & Model & $N_{\mathrm{H}}$ & $\begin{array}{c}k T \\
(\mathrm{keV})\end{array}$ & $\alpha$ & $\begin{array}{c}L \\
\left(\operatorname{erg~s}^{-1}\right)\end{array}$ & $\chi^{2} /$ dof \\
\hline \multirow[t]{4}{*}{$\mathrm{X}-4$} & Power-law & $2.0 \pm \begin{array}{l}6.5 \\
1.9\end{array}$ & $\ldots$ & $3.4 \pm \begin{array}{l}2.8 \\
1.0\end{array}$ & $1.510^{38}$ & $13.7 / 12$ \\
\hline & Power-law & 0.056 fixed & & $2.7 \pm \begin{array}{l}0.6 \\
0.4\end{array}$ & $7.910^{37}$ & $15.0 / 13$ \\
\hline & Bremsstrahlung & $<4.9$ & $1.8 \pm \begin{array}{l}1.8 \\
0.9\end{array}$ & $\ldots$ & $8.610^{37}$ & $13.2 / 12$ \\
\hline & Bremsstrahlung & 0.056 fixed & $2.3 \pm \begin{array}{l}1.4 \\
0.6\end{array}$ & $\ldots$ & $7.010^{37}$ & $13.7 / 13$ \\
\hline \multirow[t]{4}{*}{$X-5$} & Power-law & $<5.6$ & $\ldots$ & $3.2 \pm \begin{array}{l}1.2 \\
0.9\end{array}$ & $3.410^{37}$ & $10.7 / 14$ \\
\hline & Power-law & 0.056 fixed & & $2.6 \pm 0.4$ & $2.710^{37}$ & $11.6 / 15$ \\
\hline & Bremsstrahlung & $<2.9$ & $2.9 \pm \begin{array}{l}1.5 \\
1.2\end{array}$ & $\ldots$ & $2.610^{37}$ & $9.7 / 14$ \\
\hline & Bremsstrahlung & 0.056 fixed & $2.9 \pm{ }_{0.7}^{1.7}$ & $\ldots$ & $2.510^{37}$ & $9.7 / 13$ \\
\hline \multirow[t]{6}{*}{$\mathrm{X}-6$} & Power-law & $0.45 \pm{ }_{0.32}^{0.70}$ & $\ldots$ & $2.1 \pm \begin{array}{l}0.2 \\
0.3\end{array}$ & $7.610^{37}$ & $51.7 / 28$ \\
\hline & Power-law & 0.056 fixed & & $1.8 \pm \begin{array}{l}0.1 \\
0.1\end{array}$ & $7.510^{37}$ & $56.9 / 29$ \\
\hline & Bremsstrahlung & $<0.69$ & $5.6 \pm \begin{array}{l}2.5 \\
1.3\end{array}$ & $\ldots$ & $7.110^{37}$ & $44.2 / 28$ \\
\hline & Bremsstrahlung & 0.056 fixed & $6.4 \pm 2.2$ & . & $7.110^{37}$ & $45.0 / 29$ \\
\hline & Blackbody & $<0.19$ & $1.0 \pm 0.10$ & $\ldots$ & $7.110^{37}$ & $41.4 / 28$ \\
\hline & Disk-blackbody & $<0.29$ & $1.7 \pm 0.2$ & $\ldots$ & $6.810^{37}$ & $37.2 / 28$ \\
\hline \multirow[t]{4}{*}{$\mathrm{X}-7$} & Power-law & $<9.8$ & & $2.9 \pm \begin{array}{l}1.7 \\
1.3\end{array}$ & $2.810^{37}$ & $6.2 / 9$ \\
\hline & Power-law & 0.056 fixed & & $1.7 \pm \begin{array}{l}0.6 \\
0.6\end{array}$ & $1.610^{37}$ & $7.3 / 10$ \\
\hline & Bremsstrahlung & $<11$ & $3.7 \pm{ }_{2.4}^{97}$ & $\ldots$ & $1.810^{37}$ & $6.1 / 9$ \\
\hline & Bremsstrahlung & 0.056 fixed & $>3.7$ & $\ldots$ & $1.410^{37}$ & $6.9 / 10$ \\
\hline \multirow[t]{4}{*}{$\mathrm{X}-9$} & Power-law & $<3.9$ & $\ldots$ & $1.3 \pm{ }_{0.8}^{2.3}$ & $6.110^{37}$ & $7.2 / 8$ \\
\hline & Power-law & 0.056 fixed & $\ldots$ & $1.1 \pm \begin{array}{l}1.0 \\
0.6\end{array}$ & $6.810^{37}$ & $7.5 / 9$ \\
\hline & Bremsstrahlung & $<4.2$ & $>2.6$ & & $6.110^{37}$ & $7.2 / 8$ \\
\hline & Bremsstrahlung & 0.056 fixed & $>5.3$ & $\ldots$ & $5.710^{37}$ & $7.2 / 9$ \\
\hline \multirow[t]{4}{*}{$\mathrm{X}-10$} & Power-law & $<3.3$ & $\ldots$ & $1.9 \pm 0.8$ & $1.410^{38}$ & $31.2 / 18$ \\
\hline & Power-law & 0.056 fixed & $\ldots$ & $1.7 \pm \begin{array}{l}0.4 \\
0.3\end{array}$ & $1.410^{38}$ & $31.9 / 19$ \\
\hline & Bremsstrahlung & $<2.0$ & $8.7 \pm{ }_{4.6}^{14}$ & $\ldots$ & $1.410^{38}$ & $31.1 / 18$ \\
\hline & Bremsstrahlung & 0.056 fixed & $11 \pm{ }_{4.6}^{13}$ & $\cdots$ & $1.310^{38}$ & $31.2 / 19$ \\
\hline
\end{tabular}



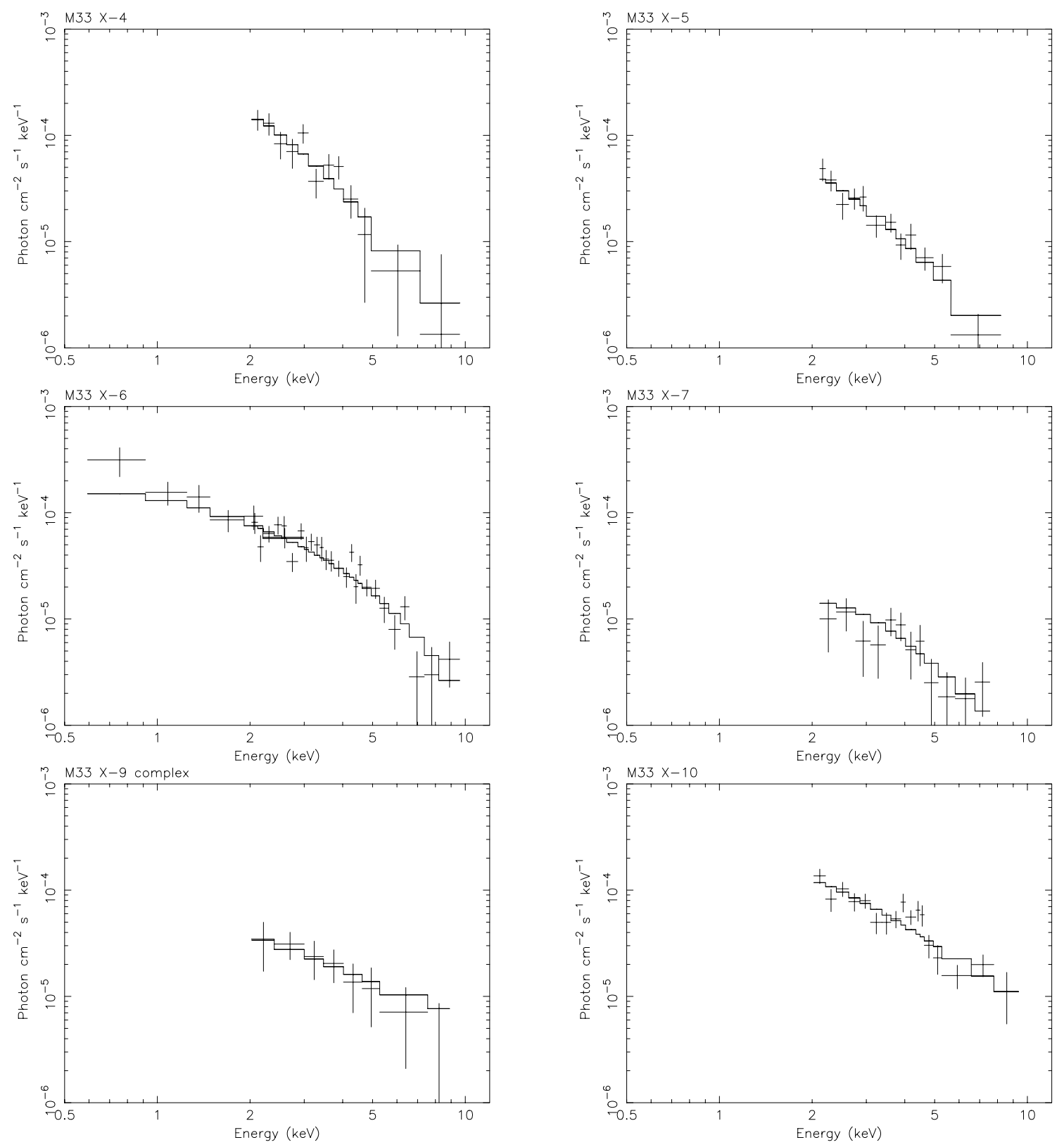

Fig. 5. MECS photon spectra of the point sources detected in the M 33 galaxy deconvolved using an absorbed power-law model except M 33 X-6 where the LECS spectrum is included and an absorbed disk-blackbody model is used. Data from all 3 observations are included, except for M 33 X-4 where the 1998 August observation is excluded. The best-fit parameters are given in Table 5. The plots all have the same extrema to aid comparison

the (hard to quantify) systematic uncertainties, we regard the detection of variability for these sources as tentative.

Since M 33 X-4 may be associated with a SNR, it is interesting to compare BeppoSAX fluxes with those obtained using Einstein and ROSAT using a bremsstrahlung model. If $N_{\mathrm{H}}$ is again fixed at $10^{21}$ atom $\mathrm{cm}^{-2}$, we obtain $k T=2.4 \mathrm{keV}$ for the MECS spectrum and $0.1-2.4 \mathrm{keV}$ and $0.2-4.5 \mathrm{keV}$ luminosities of $1.110^{38} \mathrm{ergs} \mathrm{s}^{-1}$ and $1.510^{38} \mathrm{ergs} \mathrm{s}^{-1}$, respectively. These values should be compared with $9.510^{37} \operatorname{ergss}^{-1}$ (Einstein IPC, $0.2-4.5 \mathrm{keV}$ ) and $8.310^{37} \mathrm{ergss}^{-1}$ (ROSAT, 0.1-2.4 keV), derived using this same spectral shape. Thus, there is no strong evidence for flux variability from M 33 X-4.

\section{Discussion}

The nearby galaxy M 33 was observed by the imaging X-ray instruments on-board BeppoSAX. As well as the central M $33 \mathrm{X}-8$ source which dominates the overall X-ray flux, M 33 X-4, X-5, X-6, X-7, X-9 and X-10 were clearly detected. Observations close to the expected maximum and minimum of the 105.9 day intensity cycle of M 33 X-8 failed to reveal the expected modulation, suggesting that it is probably transitory. No obvious 


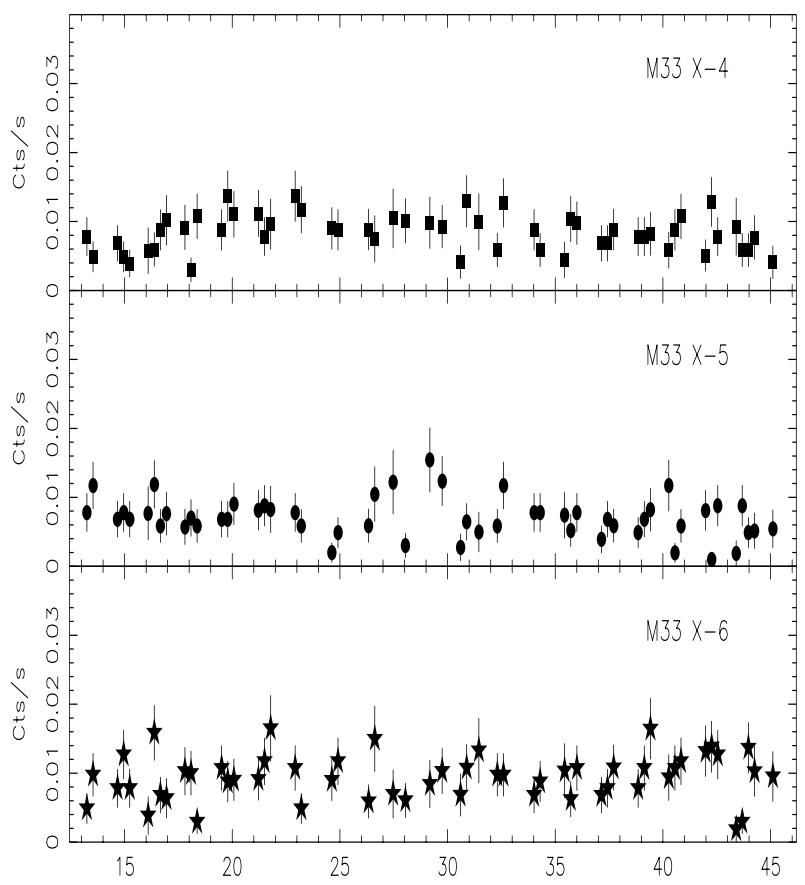

$\operatorname{Mi3} \times-7$

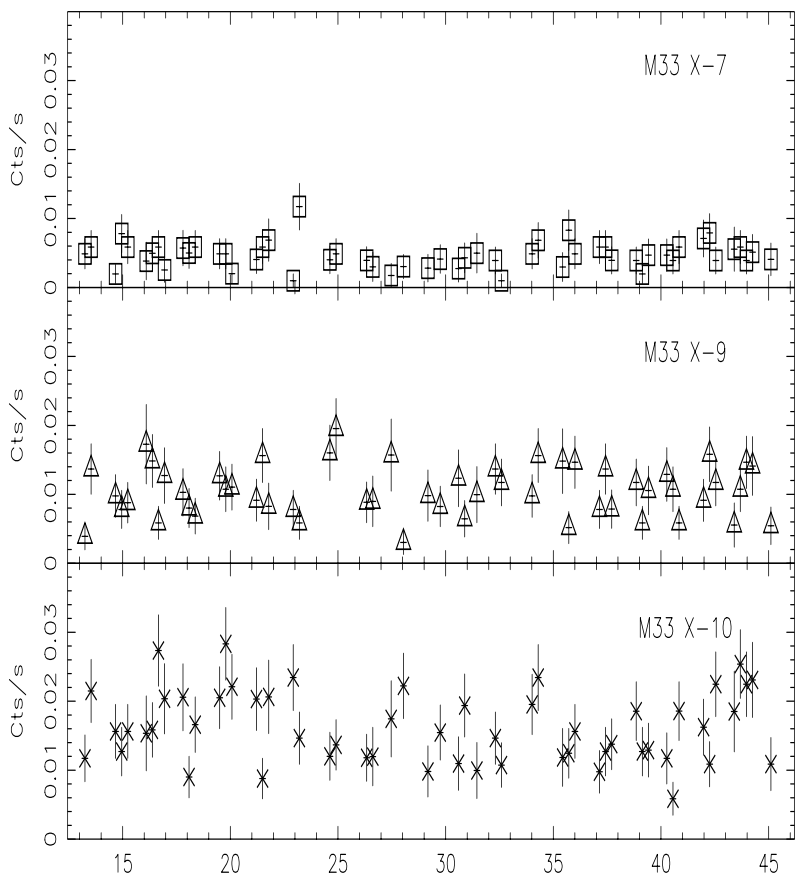

Time (hrs) of 1998 Aug 24

Fig. 6. Lightcurves for the 6 faint point sources during the 1998 August observation. No background has been subtracted

M33 Obs. 3

Bin time: 1024.

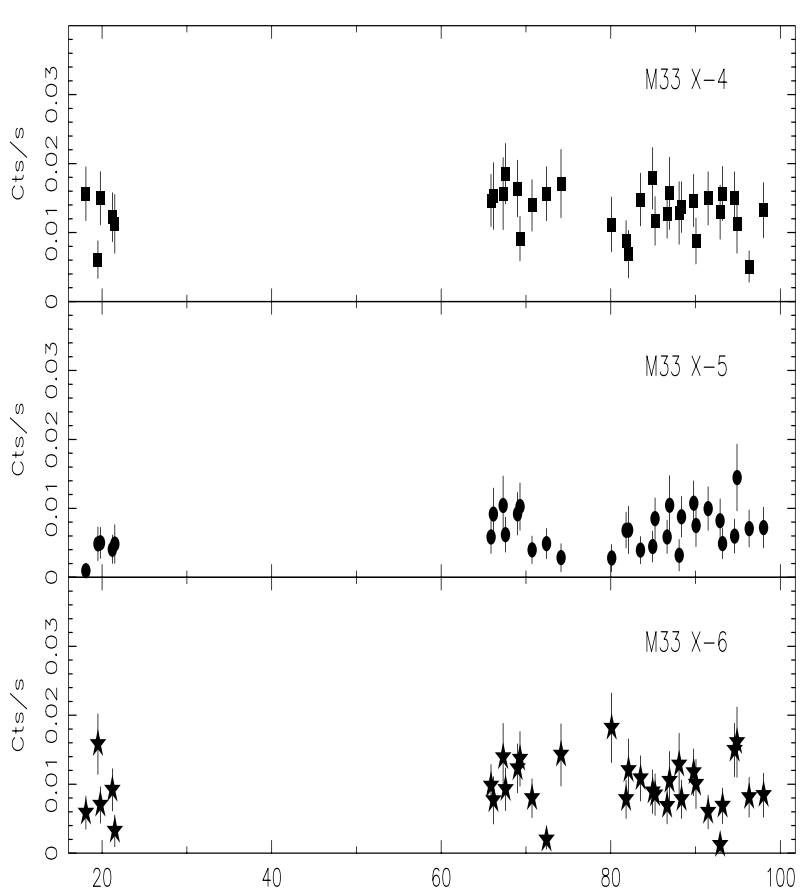

Time (hrs) of 2000 Jul 9
M33 Obs. 3

Bin time: $\quad 1024$. s

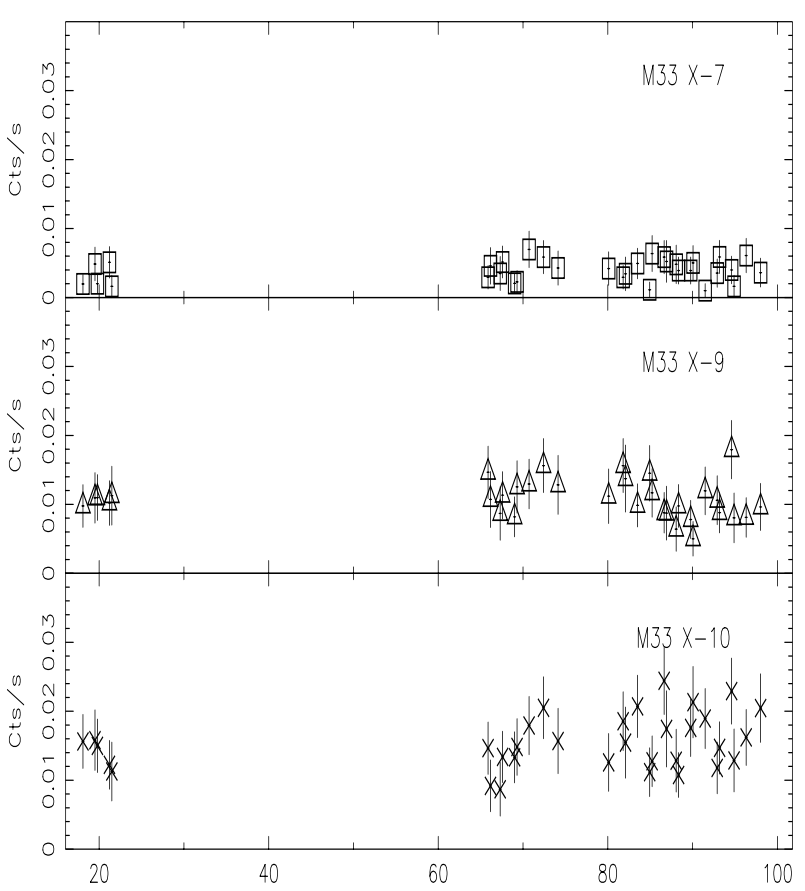

Time (hrs) of 2000 Jul 9

Fig. 7. Lightcurves for the 6 faint point sources during the 2000 July observation. No background has been subtracted 
Table 6. Comparison of the Einstein IPC and ROSAT PSPC, luminosities with those derived by extrapolating the BeppoSAX values to the Einstein $(0.2-4.5 \mathrm{keV})$ and ROSAT $(0.1-2.4 \mathrm{keV})$ energy ranges. See the text for details of the assumed spectrum. The absorption corrected luminosity, $L$, assumes a distance of $795 \mathrm{kpc}$ and is in units of $10^{37} \mathrm{erg} \mathrm{s}^{-1}$

\begin{tabular}{lcccc}
\hline Source & \multicolumn{2}{c}{$L(0.1-2.4 \mathrm{keV})$} & \multicolumn{2}{c}{$L(0.2-4.5 \mathrm{keV})$} \\
& BeppoSAX & ROSAT & BeppoSAX & Einstein \\
\hline X-4 & 18.9 & 11.1 & 18.6 & 12.4 \\
X-5 & 5.80 & 5.72 & 5.71 & 6.84 \\
X-6 & 14.0 & 18.2 & 8.57 & 16.4 \\
X-7 & 2.8 & 12.3 & 2.79 & 9.00 \\
X-9 & 8.6 & $\ldots$ & 8.57 & $\ldots$ \\
X-9(a) & $\ldots$ & 1.7 & $\ldots$ & 1.80 \\
X-9(b) & $\ldots$ & 1.9 & $\ldots$ & 0.67 \\
X-9(c) & $\ldots$ & - & $\ldots$ & 0.66 \\
X-10 & 29.0 & 18.4 & 23.6 & 16.6 \\
\hline
\end{tabular}

spectral differences between the observations are present. The $0.2-10 \mathrm{keV}$ spectrum of M $33 \mathrm{X}-8$ can best be modeled by an absorbed power-law with $\alpha=1.89 \pm{ }_{0.79}^{0.40}$ and a disk-blackbody with $k T=1.10 \pm 0.05 \mathrm{keV}$ and a projected inner-disk radius of $55.4 \pm{ }_{7.7}^{6.0} \mathrm{~km}$. This spectral shape is in good agreement with the ASCA results of Takano et al. (1994) and corresponds to a black hole mass of $\sim 10 M_{\odot}$.

The nature of M $33 \mathrm{X}-8$ is intriguing. X-ray transients such as Aql X-1 and 4U 1630-47 show outbursts on timescales of hundreds of days, but are not periodic (e.g., Kuulkers et al. 1997). A number of persistent X-ray sources exhibit long term periodicities in their $\mathrm{X}$-ray intensities. Perhaps the best known of these are the pulsars Her X-1 (35 days, Giacconi et al. 1973), LMC X4 (30.5 days, Lang et al. 1981) and SMC X-1 (60 days, Wojdowski et al. 1998). In addition, the high-mass X-ray binary SS 433 thought to contain a black hole exhibits a 164 day photometric and spectroscopic modulation in the optical band (Margon et al. 1979). Two other black hole candidates, Cyg X-1 (300 days, Priedhorsky et al. 1983 and LMC X-3, (198 or 99 days, Cowley et al. 1991) also exhibit long term X-ray modulations. Thus, the presence of a 105.9 day X-ray intensity modulation from M 33 X-8 is not particularly unusual if the source does indeed contain a $\sim 10 M_{\odot}$ black hole.

Recently, extended observations using the ASM on R$\mathrm{XTE}$ have revealed that in at least four sources (Cyg X-3, LMC X-3, SMC X-1, and GX 354-0; Paul et al. 2000; Wojdowski et al. 1998; Kong et al. 1998) the long term modulations have disappeared, or changed periods. Such unstable behavior is difficult to reconcile with the preferred model for the modulations in the high-mass sources which is obscuration by a precessing accretion disk (see e.g., Priedhorsky \& Holt 1987). Since the detection of changes in the properties of these long term variations requires very long observing baselines, it is possible that such unstable behavior is relatively common in accreting systems. Thus, the probable cessation of the 105.9 day periodicity reported here strengthens somewhat the idea that M 33 X-8 consists of a black hole accreting from a binary companion.

The 2-10 keV spectra of M 33 X-4, X-5, X-7, X-9 and $\mathrm{X}-10$ are all consistent with power-law or bremsstrahlung models, whilst that of X-6 appears to be significantly more complex, and may be reasonably well modeled with a diskblackbody with $k T=1.7 \pm 0.2 \mathrm{keV}$ and a projected innerdisk radius of $7 \pm 2 \mathrm{~km}$. This normalization is in good agreement with the average of a number of low-mass X-ray binary systems known to contain neutron stars $(\sim 7 \mathrm{~km})$, but is smaller than the average $(\sim 25 \mathrm{~km})$ for black hole candidates (e.g., Tanaka \& Lewin 1995). This suggests that M 33 X-6 may be a neutron star accreting from a low-mass companion. The spectrum of M 33 X-9 is quite hard with $\alpha=1.3$, similar to the hard/low state in galactic black hole candidates. A comparison with earlier Einstein and ROSAT results implies that $\mathrm{M} 33 \mathrm{X}-7$ and $\mathrm{X}-9$ are variable on long timescales, M $33 \mathrm{X}-4$, X-6 and X-10 may be variable, whilst X-5 does not show evidence for any such variability. We cannot exclude the association of M 33 X-4 with the supernova remnant SNR 136+396 discussed in Long et al. (1996). The lack of variability, moderate luminosity (3 $10^{37} \mathrm{erg} \mathrm{s}^{-1}$ ), and simple (consistent with a power-law or bremsstrahlung) spectrum may indicate that M $33 \mathrm{X}-5$ is a young X-ray bright supernova remnant. M 33 X-6 clearly has an unusual spectrum and is worthy of further study with more sensitive instruments. It is unsurprising that M $33 \mathrm{X}-7$ shows strong long term variability since it is known to be an (eclipsing) X-ray binary system (Peres et al. 1989). Whilst M 33 X-9 was a factor $\sim 2.5$ brighter during the BeppoSAX observations than previously observed, it is unclear which (if any) of the 3 sources that apparently comprise X-9 had become significantly brighter.

Acknowledgements. The BeppoSAX satellite is a joint ItalianDutch programme. L. Sidoli acknowledges an ESA Research Fellowship. We thank the staff of the BeppoSAX Science Data Center for help with scheduling these observations and M. Corcoran for assistance with the ROSAT analysis. The referee, H. L. Marshall, is thanked for helpful comments.

\section{References}

Boella, G., Chiappetti, L., Conti, G., et al. 1997, A\&AS, 122, 327

Boulesteix, J., Courtes, G., Laval, A., et al. 1974, A\&A, 37, 33

Christian, C. A., \& Schommer, R. A. 1982a, ApJ, 253, L13

Christian, C. A., \& Schommer, R. A. 1982b, ApJS, 49, 405

Cowley, A. P., Schmidtke, P. C., Ebisawa, K., et al. 1991, ApJ, 381,526

Dickey, J. M., \& Lockman, F. J. 1990, ARA\&A, 28, 215

Dubus, G., Charles, P. A., Long, K. S., \& Hakala, P. J. 1997, ApJ, 490, L47

Dubus, G., Charles, P. A., Long, K. S., Hakala, P. J., \& Kuulkers, E. 1999, MNRAS, 302, 731

Frontera, F., Costa, E., Dal Fiume, D., et al. 1997, A\&AS, 122, 371 
Giacconi, R., Gursky, H., Kellogg, E., et al. 1973, ApJ, 184, 227

Gottwald, M., Pietsch, W., \& Hasinger, G. 1987, A\&A, 175, 45

Haberl, F., \& Pietsch, W. 2000, in The Interstellar Medium in M 31 and M 33, ed. E. M. Berkhuijsen, R. Beck, \& R. A. M. Walterbos (Aachen, Shaker Verlag)

Kong, A. K. H., Charles, P. A., \& Kuulkers, E. 1998, New Astron., 3, 301

Kuulkers, E., Parmar, A. N., Kitamoto, S., Cominsky, L. R., \& Sood, R. K. 1997, MNRAS, 291, 81

Lang, F. L., Levine, A. M., Bautz, M., et al. 1981, ApJ, 246, L21

Larson, D. T., \& Schulman, E. 1997, AJ, 113, 618

Long, K. S., D'Odorico, S., Charles, P. A., \& Dopita, M. A. 1981, ApJ, 246, L61

Long, K. S., Charles, P. A., Blair, W. P., \& Gordon, S. M. 1996, ApJ, 466, 750

Makishima, K., Maejima, Y., Mitsuda, K., et al. 1986, ApJ, 285,712

Markert, T. H., \& Rallis, A. D. 1983, ApJ, 275, 571

Mitsuda, K., Inoue, H., Koyama, K., et al. 1984, PASJ, 36, 741

Margon, B., Grandi, S. A., Stone, R. P. S., \& Ford, H. C. 1979, ApJ, 233, L63
Morrison, D., \& McCammon, D. 1983, ApJ, 270, 119

Parmar, A. N., Martin, D. D. E., Bavdaz, M., et al. 1997, A\&AS, 122, 309

Paul, B., Kitamoto, S., \& Makino, F. 2000, ApJ, 528, 410

Peres, G., Reale, F., Collura, A., \& Fabbiano, G. 1989, ApJ, 336,140

Priedhorsky, W. C., \& Holt, S. S. 1987, Space Sci. Rev., 45, 291

Priedhorsky, W. C., Terrell, J., \& Holt, S. S. 1983, ApJ, 270 233

Schulman, E., Bregman, J. N., Collura, A., Reale, F., \& Peres, G. 1993, ApJ, 418, L67

Schulman, E., Bregman, J. N., Collura, A., Reale, F., \& Peres, G. 1994, ApJ, 426, L55

Sunyaev, R. A., \& Trümper, J. 1979, Nature, 279, 506

Takano, M., Mitsuda, K., Fukazawa, Y., \& Nagase, F. 1994, ApJ, 436, L47

Tanaka, K., \& Lewin, W. H. G. 1995, in X-ray Binaries, ed. W. H. G. Lewin, J. van Paradijs, \& E. P. J. van den Heuvel (Cambridge, Cambridge Univ. Press), 121

Trinchieri, G., Fabbiano, G., \& Peres, G. 1988, ApJ, 325, 531

Van den Bergh, S. 1991, PASP, 105, 609

Wojdowski, P., Clark, G. W., Levine, A., et al. 1998, ApJ, 502, 253 